\title{
INTRODUCTION À... \\ La spectroscopie Raman classique et à la diffusion Raman exaltée de surface
}

\author{
Nordin FELIDJ \\ Université Paris Diderot, \\ Sorbonne Paris Cité, \\ CNRS UMR 7086, \\ 15 rue Jean-Antoine de Baï, \\ 75205 Paris Cedex 13, France \\ nordin.felidj@univ-paris-diderot.fr
}

\begin{abstract}
Parmi les méthodes analytiques instrumentales, les techniques de spectroscopie vibrationnelle se sont imposées depuis longtemps. Elles permettent d'identifier la composition chimique de substances et de les quantifier. Parmi ces techniques, la spectroscopie Raman exploite un effet qui tient son nom d'un physicien indien, Sir Raman, qui, le premier en 1928, mit en évidence ce phénomène (il obtint le prix Nobel pour cela en 1930) [1].
\end{abstract}

source laser), les photons constituant cette radiation peuvent être transmis, absorbés ou diffusés dans toutes les directions de l'espace. L'analyse spectroscopique de la lumière diffusée montre que l'essentiel des photons réémis ont la même fréquence $v_{0}$ que le rayonnement incident. Cette diffusion sans changement de fréquence est appelée diffusion Rayleigh (ou diffusion élastique). Pour moins d'un million de photons diffusés, un changement de fréquence est observé: cette diffusion inélastique de la lumière constitue l'effet Raman. Les fréquences des photons diffusés de façon inélastique valent $v_{\mathrm{RS}}=v_{0}-v_{\mathrm{v}}$ et $v_{\mathrm{RAS}}=v_{0}+v_{\mathrm{v}}$ (où $v_{\mathrm{V}}$ représente la fréquence d'un mode de vibration de la molécule). On parlera ainsi de diffusion Raman Stokes et Raman anti-Stokes pour les fréquences respectives $v_{\text {RS }}$ et $v_{\text {RAS }}$. Comme en spectroscopie IR, on indique la position des raies Raman en nombre d'onde $(\bar{v}=1 / \lambda)$ exprimé en $\mathrm{cm}^{-1}$. De plus, plutôt que de repérer ces raies par le nombre d'onde absolu, on préferera faire coïncider le zéro de l'échelle horizontale avec le nombre d'onde de la raie excitatrice (voir figure 1).
On remarquera aussi que l'intensité des raies Stokes, détectées vers les plus basses fréquences, est toujours supérieure à celle des raies anti-Stokes, comme illustré sur le spectre de la figure 1. Il est d'usage de compter positivement l'échelle des nombres d'onde des spectres Raman pour les raies Stokes, compte tenu de leur plus grande intensité.

\section{Description élémentaire de l'effet Raman}

L'appréhension du phénomène de diffusion Raman fait intervenir la polarisabilité $\alpha$. Cette grandeur exprime la faculté du nuage électronique d'un système moléculaire à se déformer sous l'effet d'un champ électrique $\mathrm{E}_{0}$, pour acquérir un moment dipolaire induit $\mathrm{P}: \mathrm{P}=\alpha \mathrm{E}_{0}$ dans le cas d'une molécule à symétrie sphérique. En fait, $\alpha$ n'est pas un simple coefficient de proportionnalité, mais un tenseur de rang 2 .

Si le champ $\mathrm{E}_{0}$ oscille à la fréquence $v_{0}$, le moment dipolaire induit oscille également à cette fréquence et rayonne un champ électrique. Il s'agit de la diffusion Rayleigh. De plus, la 
polarisabilité $\alpha$ fluctue également au cours des vibrations de la molécule. Autrement dit, la déformation du nuage électronique sous l'effet d'un champ électrique $E_{0}$ est modulée par les mouvements de vibration. Par conséquent, outre la diffusion Rayleigh à la fréquence $v_{0}$, les modes de vibration qui modulent la polarisabilité sont à l'origine d'un rayonnement supplémentaire aux fréquences $v_{\mathrm{RS}}=v_{0}-v_{\mathrm{v}}$ et $v_{\mathrm{RAS}}=v_{0}+v_{\mathrm{v}}$ (où $v_{\mathrm{v}}$ représente la fréquence d'un mode de vibration de la molécule). Ce traitement classique reproduit bien les observations expérimentales en termes de position des raies Rayleigh et Raman, mais non les intensités relatives correctes concernant les raies Stokes et anti-Stokes (figure 2a).

Pour rendre compte de la différence d'intensité des raies Raman Stokes et anti-Stokes, il est nécessaire de faire intervenir la quantification des niveaux d'énergie de la molécule (figure $2 b$ ). Ainsi, dans le cadre d'une description quantique, on peut montrer que le rapport des intensités Raman Stokes et anti-Stokes est proportionnel au rapport des populations des niveaux vibrationnels, qui obéit à une loi de Boltzmann. Une conséquence de cette loi est que l'intensité Stokes est systématiquement inférieure à l'intensité anti-Stokes, en accord avec les observations expérimentales (voir la figure 1).

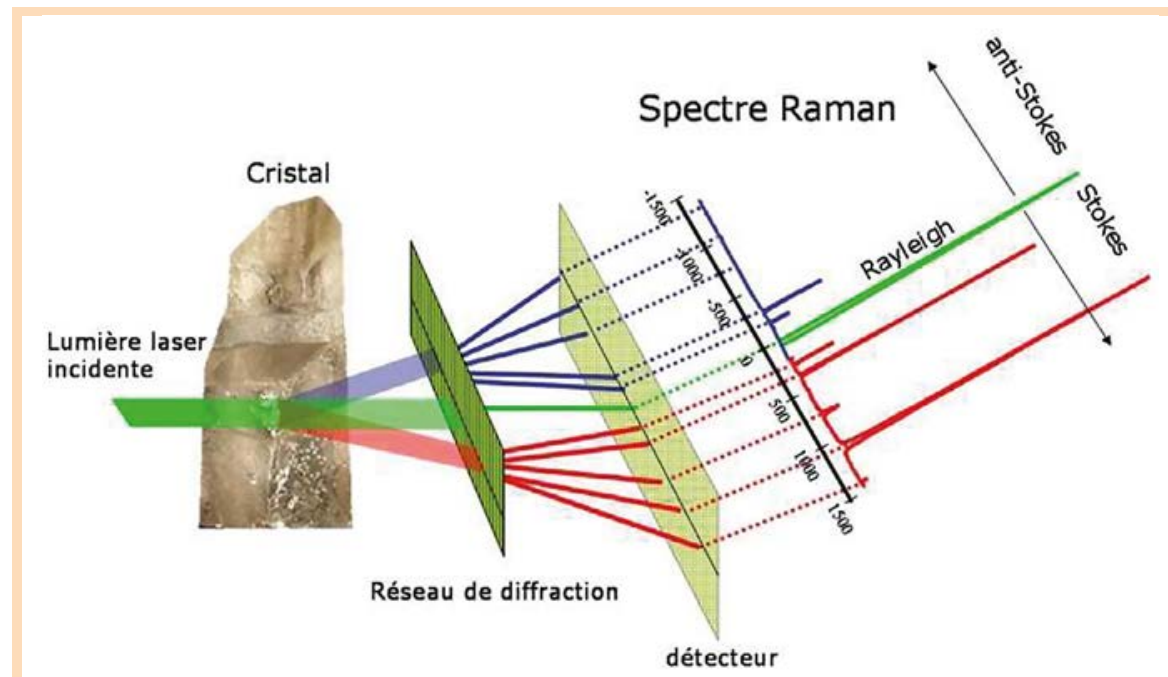

Figure 1. Principe de l'effet Raman. (Crédit photo: Philippe Gillet, 2004)

\section{Règles de sélection et symétrie des vibrations}

Tous les modes de vibration d'une molécule ne sont pas observables en diffusion Raman. Il existe en effet des règles de sélection qui établissent les critères d'activité des raies Raman. L'analyse des vibrations des molécules, ainsi que la détermination de leur activité en Raman nécessite de faire intervenir les propriétés de symétrie de la molécule. L'application des résultats connus de la théorie des groupes apporte ainsi une solution élégante à la prévision du nombre de modes de vibration et de leur activité. On peut ainsi montrer qu'un mode de vibration $Q$ sera actif en Raman si $Q$ se transforme comme l'une au moins des composantes de la polarisabilité $\alpha$, données dans la table de caractère du groupe de symétrie de la molécule. Voici quelques règles permettant de prévoir l'activité Raman et IR.

- Si la molécule possède un centre de symétrie, il n'existe aucune vibration commune aux spectres IR et Raman. Les vibrations symétriques par rapport à ce centre sont actives en Raman, alors que les vibrations antisymétriques sont actives en IR. Cette règle montrel'aspect complémentaire des spectrométries IR et Raman, qui est exclusif lorsqu'il existe un centre de symétrie. Dans le cas contraire, si certains modes sont actifs à la fois en IR et en Raman, cela indique de façon certaine que la molécule ne possède pas de centre de symétrie.

\section{Filtres Interférentiels}

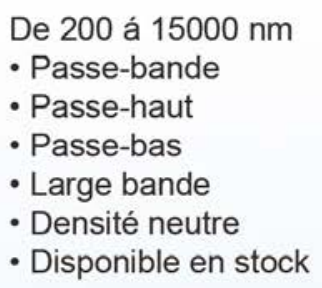

\section{Réseaux Holographiques}

De 150 á $2000 \mathrm{~nm}$

- Compression d'impulsion

- Télècom

- Accordabilité spectrale

- Monochromateurs

- Spectroscopie

- Disponible en stock



UK (parle francais): sales uk@spectrogon.com·Tel +44 1592770000 
- Si la molécule possède au moins un axe de symétrie d'ordre supérieur à deux, des modes dégénérés apparaissent. Par exemple, une dégénérescence double signifie que deux modes sont confondus en une seule raie.

- Les vibrations totalement symétriques sont toujours actives en Raman, pour tous les groupes de symétrie. Les raies correspondantes sont souvent intenses. Lorsqu'elles sont actives également en IR, elles sont par contre de faible intensité dans ce spectre. L'inverse est vrai: les raies qui sont actives en IR et Raman, et très intenses en IR, sont souvent de faible intensité dans le spectre Raman.

\section{Instrumentation}

En une trentaine d'années, l'instrumentation en spectrométrie Raman a bénéficié de progrès très importants en optique, électronique de détection et de pilotage, et informatique.

Les spectromètres Raman et microRaman confocal (analyse sous microscope) sont aujourd'hui des outils performants et d'une grande fiabilité. Quel que soit le modèle, la configuration de base se compose des éléments suivants: - Une source de lumière laser: traditionnellement, les lasers continus à argon ionisé restent les plus utilisés. Ils offrent des raies puissantes dans le visible et l'ultra-violet. Cependant, les plus abordables sont probablement les diodes lasers. Les longueurs d'onde disponibles nombreuses dans le visible
(685, 785, $810 \mathrm{~nm} . .$.$) , s'accompagnent$ de puissances pouvant aller jusqu'à $1 \mathrm{~W}$ continu. Une puissance de quelques dizaines de mW est largement suffisante pour obtenir un signal détectable. Notons enfin que la présence de nombreuses raies parasites accompagnant l'émission laser peut être gênante, et on utilise alors un filtre interférentiel devant l'échantillon pour les éliminer. - Le spectrographe: un double ou même triple monochromateur est nécessaire pour extraire le signal Raman. En effet, la diffusion Rayleigh de l'échantillon pouvant être un million de fois plus intense que l'émission Raman, la diffusion parasite de l'émission Rayleigh créée par les imperfections des réseaux de diffraction rend la détection Raman impossible. D'où la présence du double ou triple spectrographe. Le premier servira à séparer et bloquer la raie d'excitation laser, et le deuxième (puis le troisième éventuellement), à séparer spectralement la diffusion Raman des parasites générés par le premier.

- La détection: la diffusion étant déjà peu intense par nature, le signal Raman se réduit à quelques photons par seconde. Dans les montages récents, on utilise des détections dites multi-canales, avec lesquelles il est possible d'analyser simultanément un grand nombre d'éléments spectraux, très rapidement. Des détecteurs CCD (charge coupled devices) refroidis par effet Peltier $\left(-40^{\circ} \mathrm{C}\right)$ présentent un bruit extrêmement faible (équivalent à moins d'un photon par minute), et permet la détection de signaux à très bas niveau. La tête de détection multicanale étant composée d'une matrice bidimensionnelle de diodes, celle-ci est particulièrement bien adaptée pour les études en imagerie Raman. Il est ainsi possible d'accéder à la distribution de différentes espèces chimiques dans un échantillon (repérées par des pics Raman différents), avec une limite de résolution de l'ordre du micromètre.

\section{La diffusion Raman exaltée de suirface}

La spectroscopie Raman classique (ou spontanée) est une technique très peu sensible, ce qui limite l'analyse de produits à faible concentration. Néanmoins, l'adsorption de molécules à la surface de nanostructures métalliques (principalement or et argent), permet d'exalter leur signature Raman, $y$ compris à très faible concentration. On parlera de diffusion Raman exaltée de surface (DRES ou SERS en anglais, pour surface enhanced raman scattering) [2]. Cette adsorption doit se faire sur une surface rugueuse, dans une solution colloïdale ou sur des substrats de particules métalliques auto-assemblées ou lithographiées. L'exaltation des spectres Raman peut atteindre ainsi un facteur de l'ordre de $10^{7}-10^{8}$. Un autre aspect de l'effet DRES est l'extinction de la fluorescence des adsorbats. Aussi, les avantages de cette méthode facile à mettre en œuvre ont
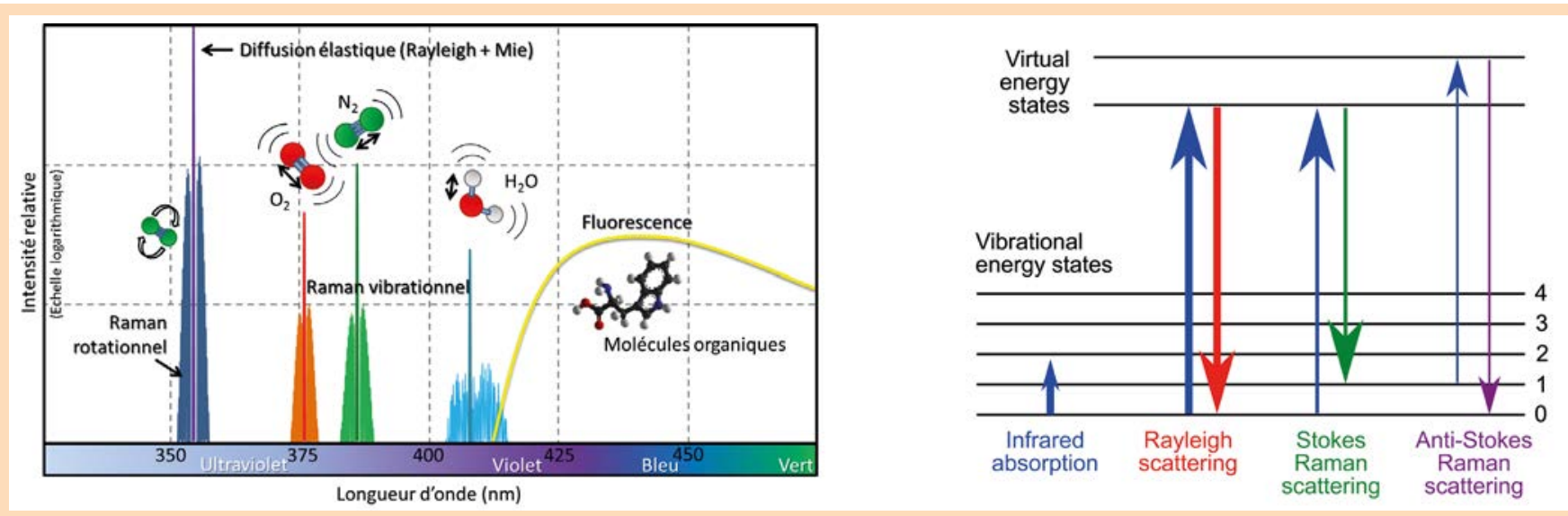

(a) Spectre de la diffusion Raman et de la fluorescence, permettant le sondage d'espèces chimiques spécifiques (crédit photo: Kilohn limahn). (b) Diagramme des niveaux d'énergie impliqués dans la spectroscopie infrarouge, la diffusion Rayleigh et la diffusion Raman. L'épaisseur des lignes indique qualitativement l'intensité des signaux de chaque transition (Creative Commons). 
$\triangleright$ Figure 3. Sous excitation laser, le moment dipolaire induit des molécules adsorbées à la surface de structures métalliques est considérablement amplifié, grâce à une exaltation du champ électrique. Cette exaltation, d'origine électromagnétique, liée à l'excitation des plasmons de surface localisés, est à l'origine de l'effet DRES.

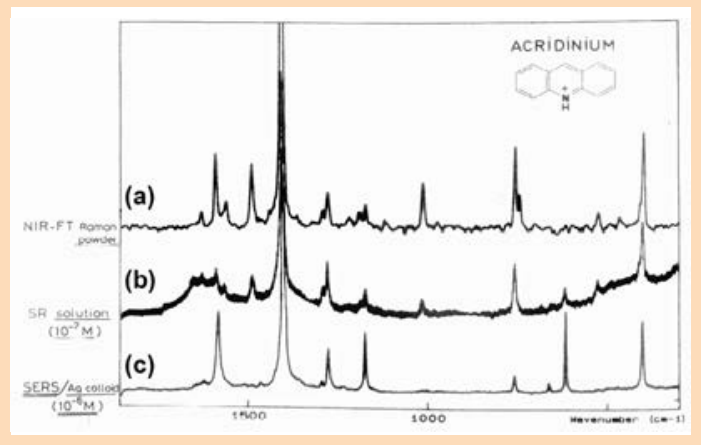

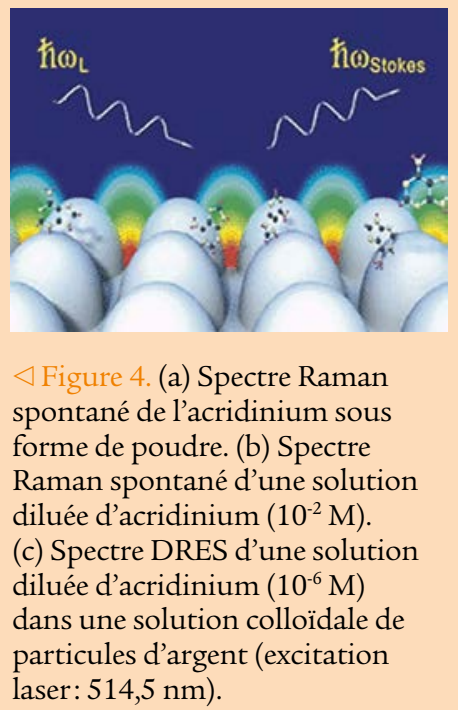

suscité de nombreuses études et des applications dans des domaines aussi variés que la médecine, la pharmacologie, la défense ou le monde de l'art.

Les premières observations d'un spectre Raman exalté de surface ont été faites au milieu des années 70 dans le cas de la pyridine adsorbée à la surface d'une électrode d'argent rendue rugueuse par des cycles d'oxydo-réduction, et en solution colloïdale d'argent agrégé [3]. L'origine de l'effet DRES est attribuée à l'amplification du champ électrique local, consécutif à l'excitation des plasmons de surface localisés (PSL). Ce phénomène optique correspond à une oscillation collective en phase des électrons de conduction à la surface de la nanostructure, sous excitation lumineuse. Ces oscillations se traduisent par une forte amplification du champ local, ressentie par la molécule adsorbée, et donc de son moment dipolaire induit (figure 3). L'exaltation des spectres Raman peut également s'expliquer par une augmentation de la polarisabilité de la molécule lors de son interaction avec la surface métallique, mais le facteur d'exaltation associé n'excède pas 100.

Pour illustrer cet effet DRES, la figure 4 montre le spectre Raman exalté de surface de la molécule d'acridinium. On constate que le spectre DRES de l'acridinium à $10^{-6} \mathrm{M}$ (ou mole par litre) est beaucoup plus intense que le spectre Raman spontané de l'acridinium à $10^{-2} \mathrm{M}$, malgré 4 ordres de grandeur en moins en terme de concentration.

\section{Conclusion}

Rarement une spectroscopie ne s'est révélée aussi riche, tant par la multiplicité de ses effets que par l'étendue du champ de ses applications. Il existe en effet d'autres effets Raman intéressants comme la diffusion Raman résonante (pour laquelle une exaltation de certaines raies est observée, à condition que la longueur d'onde laser soit voisine de celle d'une transition électronique de la molécule étudiée). À ces effets linéaires s'ajoutent des effets non linéaires observables lorsque le champ électrique créé par le rayonnement incident est très élevé (c'est le cas des lasers pulsés, avec des puissances crêtes jusqu'au gigawatt). Ces effets non linéaires ont donné naissance à des techniques telles que l'effet Raman stimulé, l'effet hyper Raman, ou la diffusion Raman anti-Stokes cohérente (DRASC, ou CARS en anglais pour coberent antiStokes Raman spectroscopy).

\section{RÉFÉRENCES}

[1] C. V. Râman, The molecular scattering 267-275 (1930)

[2] M. Moskovitz, Persistent misconceptions regarding SERS. Phys. Chem. Chem. Phys., 15, 5301 (2013)

[3] M. Fleischmann, P.J. Hendra, A.J. McQuillan, Raman spectra of pyridine adsorbed at a silver electrode, Chem. Phys. Lett. 26, 163-166 (1974) of light. Nobel Lectures: Physics, 1922-1941,

\section{aCal| bfi}

Votre partenaire en Spectroscopie

Spectroradiomètres

Radiance, Luminance, Couleurs, CRI

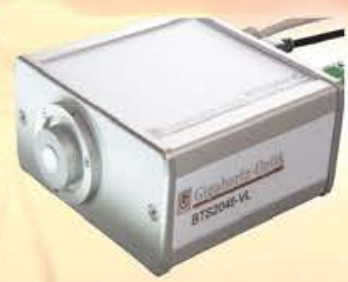

\section{Spectromètres}

Fabry-Perot, FTIR, Réseaux $0.2 \mu \mathrm{m}$ à $12 \mu \mathrm{m}$



Caméras hyperspectrales $0.18 \mu \mathrm{m}$ aे $4.2 \mu \mathrm{m}$

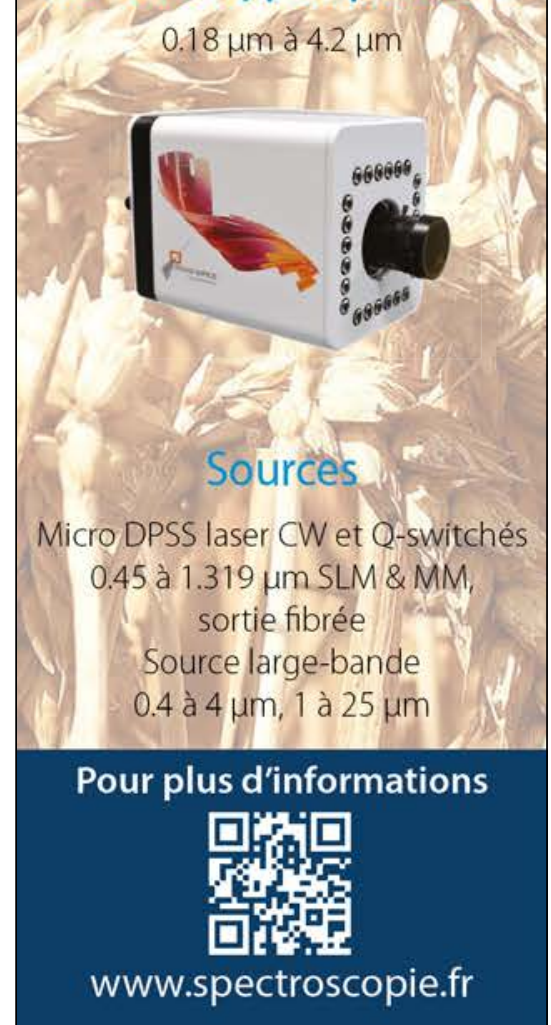

